\title{
Three-dimensional analysis of regional left ventricular endocardial curvature from cardiac magnetic resonance images
}

\author{
Francesco Maffessanti ${ }^{\mathrm{a}, \mathrm{b}}$, Roberto M. Lang ${ }^{\mathrm{a}}$, Johannes Niel ${ }^{\mathrm{c}}$, Regina Steringer-Mascherbauer ${ }^{\mathrm{c}}$, \\ Enrico G. Caiani ${ }^{b}$, Hans-Joachim Nesser ${ }^{c}$, Victor Mor-Avi ${ }^{a}$ ** \\ ${ }^{a}$ University of Chicago, Chicago, IL 60637, USA \\ ${ }^{\mathrm{b}}$ Politecnico di Milano, Milan 20133, Italy \\ ${ }^{\mathrm{c}}$ Public Hospital Elisabethinen, Linz A4010, Austria \\ Received 26 April 2010; accepted 2 November 2010
}

\begin{abstract}
Purpose: Left ventricular (LV) remodeling is usually assessed using global changes in LV volume. We hypothesized that threedimensional analysis of regional endocardial curvature from magnetic resonance images could provide clinically useful information on localized LV remodeling. We tested this approach by investigating regional differences in endocardial curvature in normal and hypokinetic ventricles.

Materials and Methods: Images were obtained in 44 patients with normal LV function (NL, $N=14)$, dilated cardiomyopathy (DCM, $N=15$ ) or ischemic heart disease (IHD, $N=15)$. Local surface curvedness, normalized to take into account instantaneous LV size $\left(C_{\mathrm{n}}\right)$, was calculated throughout the cardiac cycle and compared between segment groups: NL $(N=401)$, IHD $(N=92)$ and DCM $(N=255)$.

Results: In all normal segments, $C_{\mathrm{n}}$ gradually increased during systole and then decreased during diastole. While both maximum and minimum values of $C_{\mathrm{n}}$ were comparable in the basal and midventricular segments, they were significantly higher in the four apical segments and highest in the apical cap. In addition, percent change in $C_{\mathrm{n}}$ was higher in mid and apical compared to basal segments $(P<.05)$. At all LV levels, $C_{\mathrm{n}}$ values in DCM segments were lower $(P<.05)$ than in NL and IHD segments, which were similar. In contrast, percent change in $C_{\mathrm{n}}$ was significantly lower in both IHD and DCM segments compared to NL.

Conclusion: Three-dimensional analysis of LV endocardial curvature yielded quantitative information on regional ventricular shape consistent with the known pathophysiology, supporting its potential clinical usefulness in the evaluation of LV remodeling.
\end{abstract}

(C) 2011 Elsevier Inc. All rights reserved.

Keywords: Cardiac magnetic resonance imaging; Left ventricle; Three-dimensional analysis; Endocardial curvature; Endocardial curvedness

\section{Introduction}

Left ventricular (LV) remodeling as a result of disease progression or in response to therapy is reflected by changes in ventricular size and shape, which may be independent of each other. Nevertheless, to date, quantitative assessment of $\mathrm{LV}$ remodeling has been predominantly described in terms of changes in LV volume and ejection fraction, while disregarding the simultaneously occurring changes in ventricular shape. While a variety of techniques for quantitative evaluation of LV volume have become part of standard clinical practice [1-3], the development of tools for

* Corresponding author. Tel.: +1 773702 1842; fax: +1 7737021034 .

E-mail address: vmoravi@bsd.uchicago.edu (V. Mor-Avi). quantitative evaluation of ventricular shape has been explored only partially [4-7]. This is despite several studies indicating that LV shape analysis may be potentially useful and could be used in conjunction with different imaging modalities, including radionuclide ventriculography $[8,9]$, electron beam computed tomography [10] and ultrasound images [11-14]. Moreover, LV function has been studied both on a global and regional basis, but previous studies have focused on global LV shape, while only initial feasibility data have been published regarding local LV shape $[14,15]$. A new technique was recently proposed for global LV shape analysis from real-time three-dimensional (3D) echocardiographic images [16], which was able to demonstrate independent changes in LV volume and shape as well as to identify differences between normal and abnormal ventricles. 
In the current study, we focused on the quantification of regional endocardial curvature from dynamic $3 \mathrm{D} \mathrm{LV}$ endocardial surfaces derived from cardiac magnetic resonance (CMR) images with the rationale that such analysis could prove useful in the evaluation of LV remodeling. Initial testing of curvature analysis in a small number of patients from CMR images $[17,18]$ had demonstrated its feasibility and yielded promising results that warrant further testing and validation in larger groups of patients. Accordingly, the aims of this study were (a) to determine normal patterns of regional endocardial curvature and its changes throughout the cardiac cycle by analyzing CMR images obtained in patients with normal LV wall motion and (b) to test this technique on images obtained in patients with global and regional hypokinesis, in order to understand the effects of these conditions on regional LV shape.

\section{Methods}

\subsection{Population}

We studied 44 consecutive patients referred for CMR imaging, including 14 patients with normal to mildly abnormal LV function (NL group), 15 patients with regional wall motion abnormalities secondary to ischemic heart disease (IHD group) and 15 patients with idiopathic dilated cardiomyopathy (DCM group). Clinical characteristic of the study population are summarized in Table 1. Exclusion criteria were dyspnea precluding a 5- to 10-s breath-hold, atrial fibrillation or other cardiac arrhythmias, pacemaker or defibrillator implantation, claustrophobia and other known contraindications to CMR imaging. The protocol was approved by the Institutional Review Board, and written informed consent was obtained in all patients.

\subsection{Image acquisition}

Cardiac magnetic resonance images were obtained using a 1.5-T scanner (Siemens, MAGNETOM Sonata, Erlangen, Germany) with a phased-array cardiac coil. Electrocardiogram-gated localizing spin-echo sequences were used to identify the long axis of the heart. Steady-state free precession (true FISP) dynamic gradient-echo mode was

Table 1

Clinical characteristics of the study group subdivided NL, IHD and DCM

\begin{tabular}{llll}
\hline & NL & IHD & DCM \\
\hline$n$ & 14 & 15 & 15 \\
Gender (M/F) & $10 / 4$ & $10 / 5$ & $6 / 9$ \\
Age (years) & $51 \pm 18$ & $63 \pm 11$ & $55 \pm 14$ \\
Segments with normal/abnormal & $238 / 0$ & $163 / 92$ & $0 / 255$ \\
$\quad$ wall motion & & & \\
LV EDV (ml) & $158 \pm 44$ & $195 \pm 45$ & $260 \pm 69$ \\
LV ESV (ml) & $74 \pm 34$ & $128 \pm 41$ & $197 \pm 75$ \\
LV EF (\%) & $54 \pm 10$ & $35 \pm 9$ & $26 \pm 11$ \\
\hline
\end{tabular}

EDV: end-diastolic volume; ESV: end-systolic volume; EF: ejection fraction. then used to acquire images using retrospective electrocardiographic gating and parallel imaging techniques (mSENSE) during 5- to 10-s breath-holds with a temporal resolution of 30 frames per cardiac cycle. Cine-loops of 8mm-thick slices with 2-mm gaps and $2.0 \times 2.0-\mathrm{mm}$ in-plane spatial resolution were acquired in several planes. First, three long-axis planes representing the two-, three- and fourchamber views were acquired. Subsequently, a stack of short-axis views was obtained from just above the ventricular base to just below the apex. All images were stored digitally for off-line analysis.

\section{3. $3 D$ endocardial surface detection}

The entire set of short- and long-axis images was analyzed using prototype software (4D-LV Analysis MR, TomTec Imaging Systems, Unterschleissheim, Germany), as depicted in Fig. 1. End-diastole was identified as the first frame in the sequence and end-systole as the frame depicting the smallest LV cavity. For each of these two frames, LV endocardial boundary was manually initialized in each of the three longaxis views, while including the papillary muscles and endocardial trabeculae in the LV cavity (Fig. 1, top panels). Then, the endocardial surface was reconstructed by finding the best fit to the endocardial boundaries throughout the cardiac cycle. Subsequently, the reconstructed surface was superimposed on the original image set, and its position was adjusted frame-by-frame by an experienced investigator wherever the surface did not accurately follow the endocardium. Finally, temporal smoothing was performed to avoid temporal discontinuities. The resultant dynamic endocardial surface (Fig. 1, bottom) was exported as a connected mesh for custom analysis of LV endocardial curvature.

\subsection{D curvature analysis}

Custom software was used for analysis of regional LV endocardial curvature, as schematically depicted in Fig. 2. First, for each node on the connected mesh representing the LV endocardial surface (Fig. 2, left), a quadric polynomial function was locally fitted to approximate a smooth surface using a robust method previously described elsewhere [19]. Then, for each point, two values were calculated: maximum curvature $k_{1}$, defined as the inverse of the radius of the smallest circle that would fit into the surface at that particular point, and the curvature $k_{2}$, similarly defined in the perpendicular direction (Fig. 2, middle). Then, local 3D surface curvedness, $C$, was calculated as the root mean square value of $k_{1}$ and $k_{2}$ (Fig. 2, right) [20] and then normalized by mean instantaneous LV curvedness, calculated by averaging curvedness at all nodes on the endocardial surface. This latter step was performed to compensate for changes in LV regional shape secondary to changes in LV size. Theoretically, if the ventricle were a perfect sphere contracting symmetrically, then regional curvedness everywhere would be the same as the mean curvedness calculated from the volume, and the ratio would be 1 at all times. Thus, 


\section{ARTICLE IN PRESS}

F. Maffessanti et al. / Magnetic Resonance Imaging $x x$ (2011) xxx-xxx
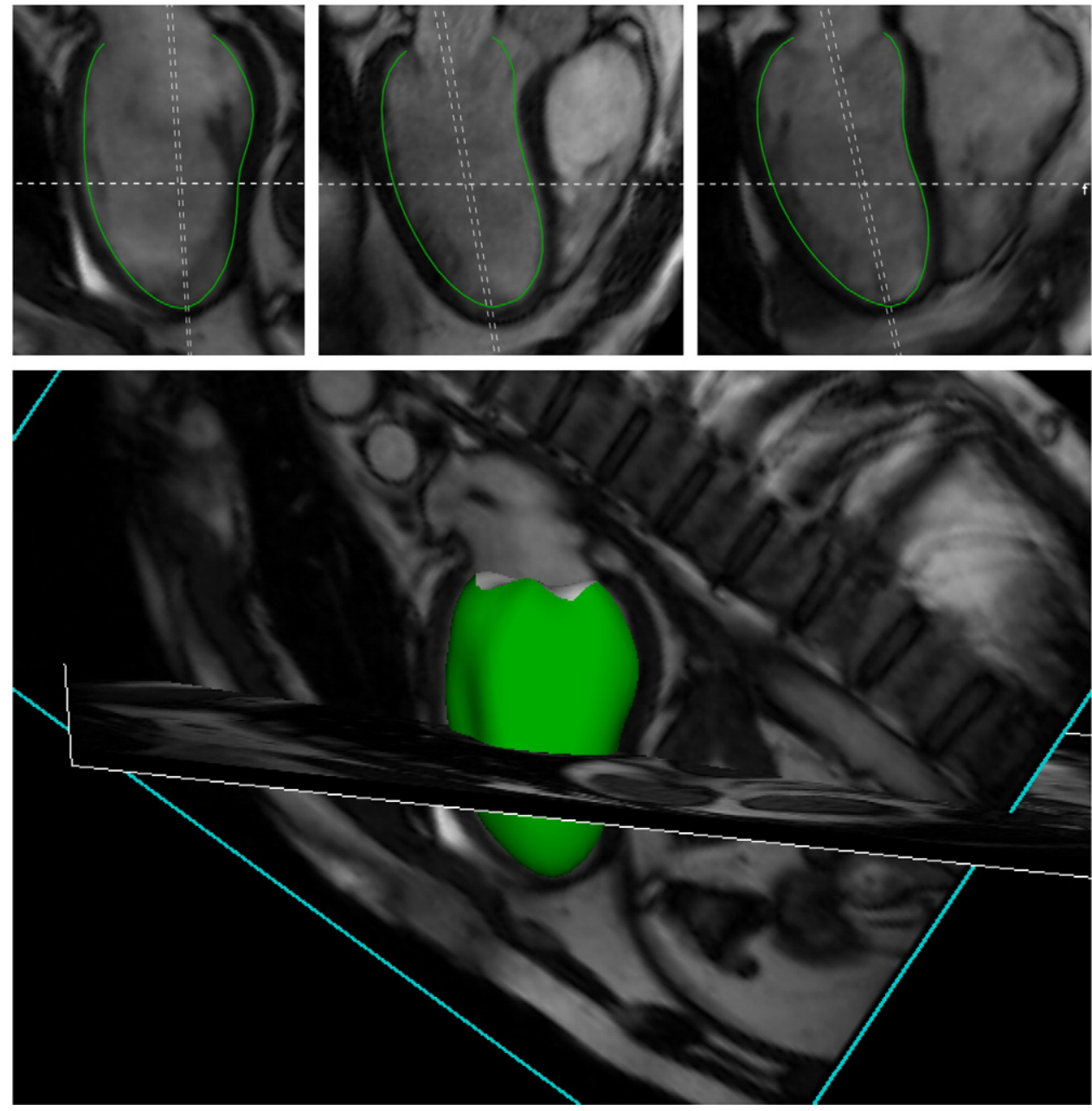

Fig. 1. Schematic representation of the 3D analysis procedure: following manual initialization of the endocardial contours at end-systole and end-diastole in three radial long-axis views (top panels from left to right: two-, three- and four-chamber views), 3D endocardial surface was reconstructed throughout the cardiac cycle and superimposed on the original images (bottom).
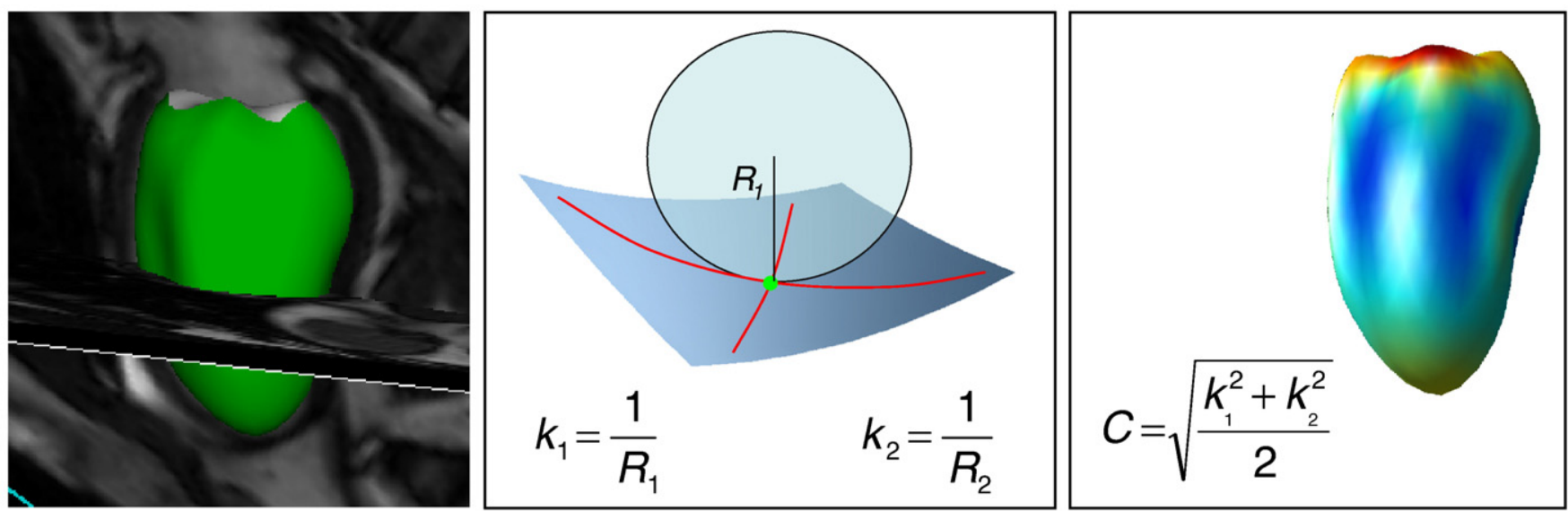

Fig. 2. Calculation of LV endocardial curvedness: for each voxel on the reconstructed endocardial surface (left), two values were calculated: maximum curvature $k_{1}$, defined as the inverse of the radius of the smallest circle that would fit into the surface at that particular point, and the curvature $k_{2}$ in the perpendicular direction (middle). Then, local 3D surface curvedness, $C$, was calculated as the root mean square value of $k_{1}$ and $\mathrm{k}_{2}$ and mapped onto the volume-rendered endocardial surface for easy visualization of curvature information (right), which was encoded with colors ranging from dark blue used for low curvedness values to dark red used for high curvedness. 
for the normalized curvedness, anything that is not 1 reflects changes in curvature beyond simple changes in the size of the left ventricle.
The normalized curvedness, $C_{\mathrm{n}}$, was mapped onto the volume-rendered endocardial surface for easy visualization of curvature information (Fig. 2, right, and video clip 1).
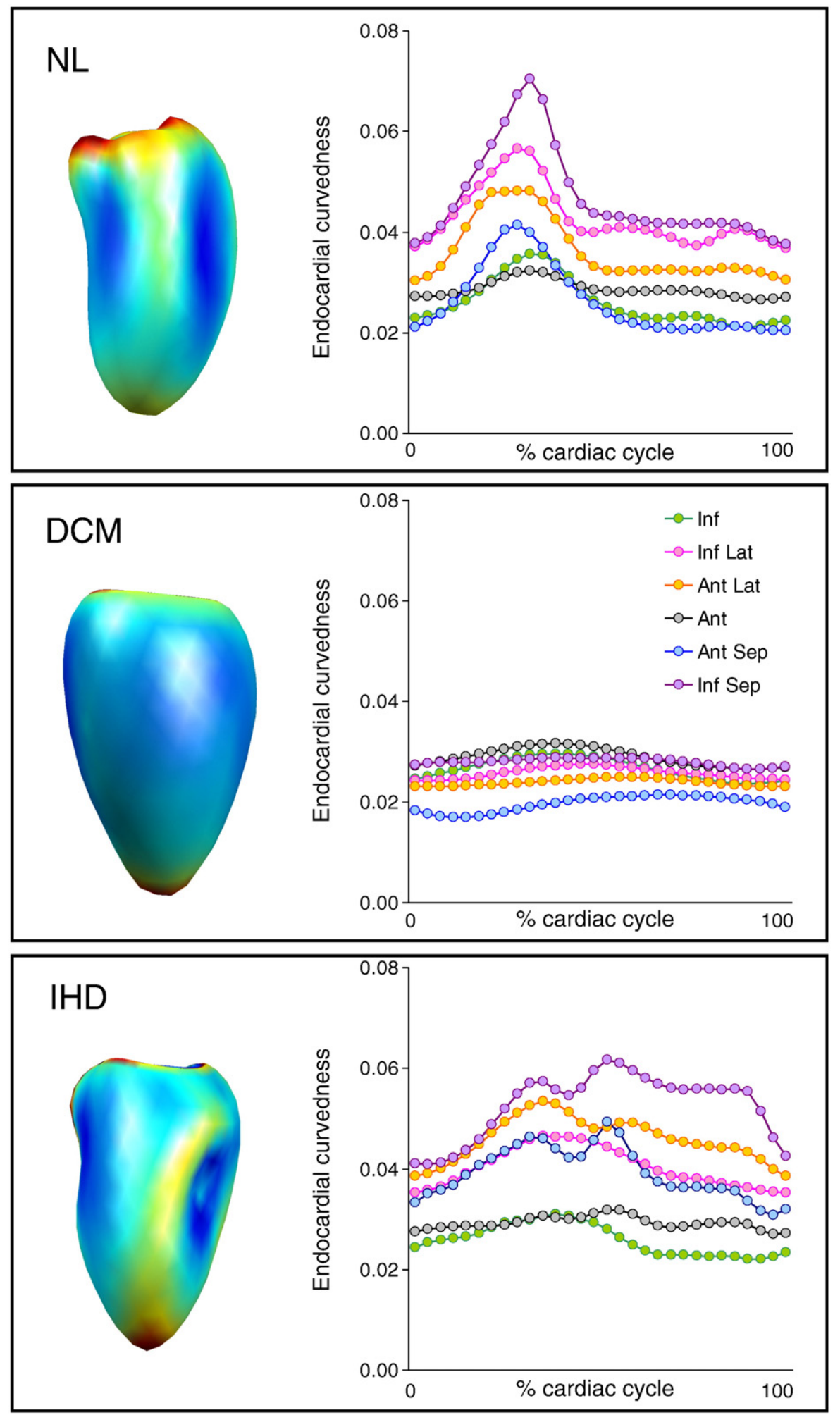

Fig. 3. Examples of LV endocardial curvedness mapped onto the volume-rendered LV endocardial surface in a patient with normal LV function (NL, top) and in two patients with wall motion abnormalities: a patient with dilated cardiomyopathy (DCM, middle) and a patient with ischemic heard disease (IHD, bottom), along with the six time-curves depicting variations in midventricular regional curvedness throughout the cardiac cycle. See text for details. 
Finally, the endocardial surface was divided into 17 segments using standard segmentation scheme recommended by the American Heart Association [21]. For each segment, regional 3D normalized curvedness was obtained by averaging local $C_{\mathrm{n}}$ values measured in all nodes in the segment.

\subsection{Segment classification}

Short-axis images at basal, midpapillary and apical levels were visually reviewed by another independent expert reader blinded to the results of 3D curvature analysis, who classified wall motion in each segment as normal or abnormal. These classifications were used to separate segments by pathology and compare endocardial curvature between NL segments at four different LV levels (basal, midventricular, apical and apical cap segments) as well as between NL and hypokinetic segments, while averaging separately segments from dilated ventricles in patients with DCM (defined as DCM segments) and abnormal segments from patients with IHD (defined as IHD segments).

\subsection{Statistical analysis}

Data were displayed in the box-plot format, wherein the box represents the first quartile, median, and third quartile values, whiskers extend from each box end to the extreme values within 1.5 times the interquartile range and outliers are shown as "+" symbols. Differences between the regional curvedness at three LV levels as well as differences in curvedness between NL, DCM and IHD segments were tested using Kruskal-Wallis test and Tukey's honestly significant difference criterion for post hoc comparisons. $P$ values $<.05$ were considered significant.

\section{Results}

Fig. 3 shows three examples of LV endocardial normalized curvedness, $C_{\mathrm{n}}$, mapped onto the respective volume-rendered $\mathrm{LV}$ endocardial surface in a patient with normal LV function and in two patients with wall motion abnormalities: a patient with DCM and a patient with IHD, along with the six time-curves depicting variations in midventricular regional curvedness throughout the cardiac cycle. In the patient with normal LV function, $C_{\mathrm{n}}$ gradually increased during systole in all segments, reaching peaks at slightly different times in different segments, and then decreased during diastole (top panel). Similar behavior can be seen in the patient with DCM, despite the considerably reduced magnitude of changes (middle panel). In contrast, in the patient with IHD, the time-curves reflected a more disorganized pattern of changes in endocardial curvedness throughout the cardiac cycle (bottom panel).
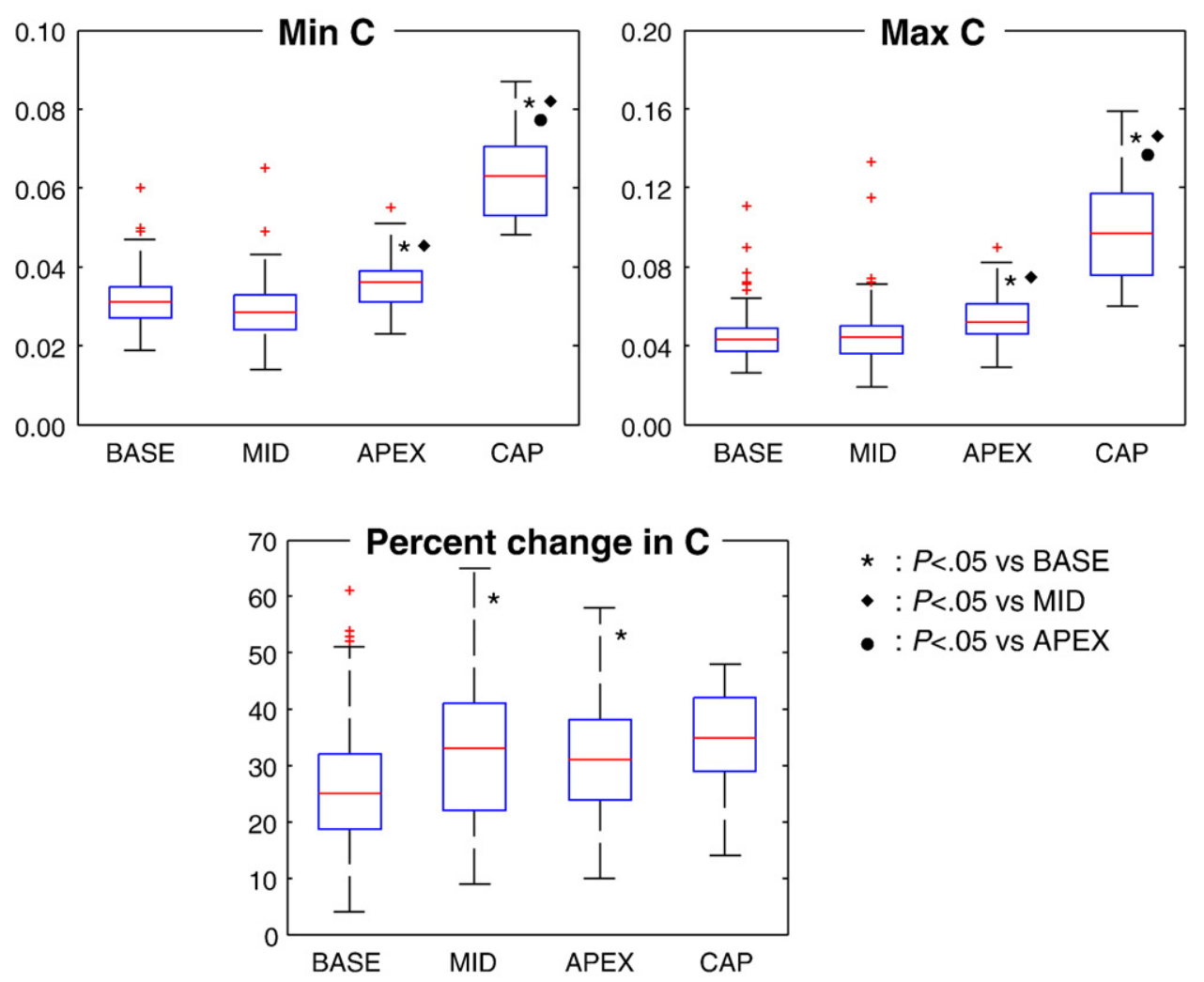

* $: P<.05$ vs BASE
- $: P<.05$ vs MID
$\bullet: P<.05$ vs APEX

Fig. 4. Box plots depicting normal variability in regional LV endocardial curvature from base to apex: minimum, maximum and percent change in regional endocardial curvedness (Min C, Max C and \% change in C) calculated for the 401 normal segments, grouped by LV level. Values expressed in $\mathrm{m}^{-1}$ for C. See text for details. 
Fig. 4 shows the summary of minimum, maximum and percent change in regional $C_{\mathrm{n}}$ for the NL segments, grouped by LV level. While both maximum and minimum values of $C_{\mathrm{n}}$ (top panels) were comparable in the basal and midventricular segments, they were significantly higher in the apical segments and highest in the apical cap. In addition, percent change in $C_{\mathrm{n}}$ (bottom) was lower in the apical region compared to basal and mid segments.

Figs. 5 through 7 show the comparisons of these three parameters between the three groups of segments: $401 \mathrm{NL}$, 92 IHD and 255 DCM segments. While minimum values of $C_{\mathrm{n}}$ were comparable between the 3 groups (Fig. 5), maximum (Fig. 6) values of regional endocardial curvedness were lower $(P<.05)$ in DCM segments than in NL and IHD segments, which were similar. In contrast, percent change in endocardial curvedness was significantly lower in both IHD and DCM segments compared to NL (Fig. 7).

\section{Discussion}

The combination of the high-contrast resolution and the dynamic nature of the steady-state free precession imaging has proven over the recent years that this imaging technique provides accurate and highly reproducible LV volume and mass measurements compared to all other cardiac imaging modalities. As a result, CMR evaluation of LV size, function and mass has become the widely accepted standard reference technique for validation of new techniques. We hypothesized that CMR images could also provide the basis for 3D evaluation of regional LV shape and deformation throughout the cardiac cycle.

In the present study, we tested the feasibility of quantifying local, LV size normalized, 3D endocardial surface curvature to describe regional LV shape and to investigate regional differences in endocardial curvature in normal and hypokinetic ventricles [17]. The potential usefulness of this approach for characterizing changes in LV shape in patients with DCM has been recently demonstrated in the context of the evaluation of the extent of scar in patients with ischemic DCM [18]. In our present study, a technique similar to that described by Garimella and Swartz [19] as robust and accurate was tested in normal ventricles to establish normal patterns of regional, sizenormalized curvature and its changes throughout the cardiac cycle, as well as under two different pathologic conditions affecting LV shape, one global (DCM) and one more localized (IHD).

\section{$\underline{\text { Min C }}$}
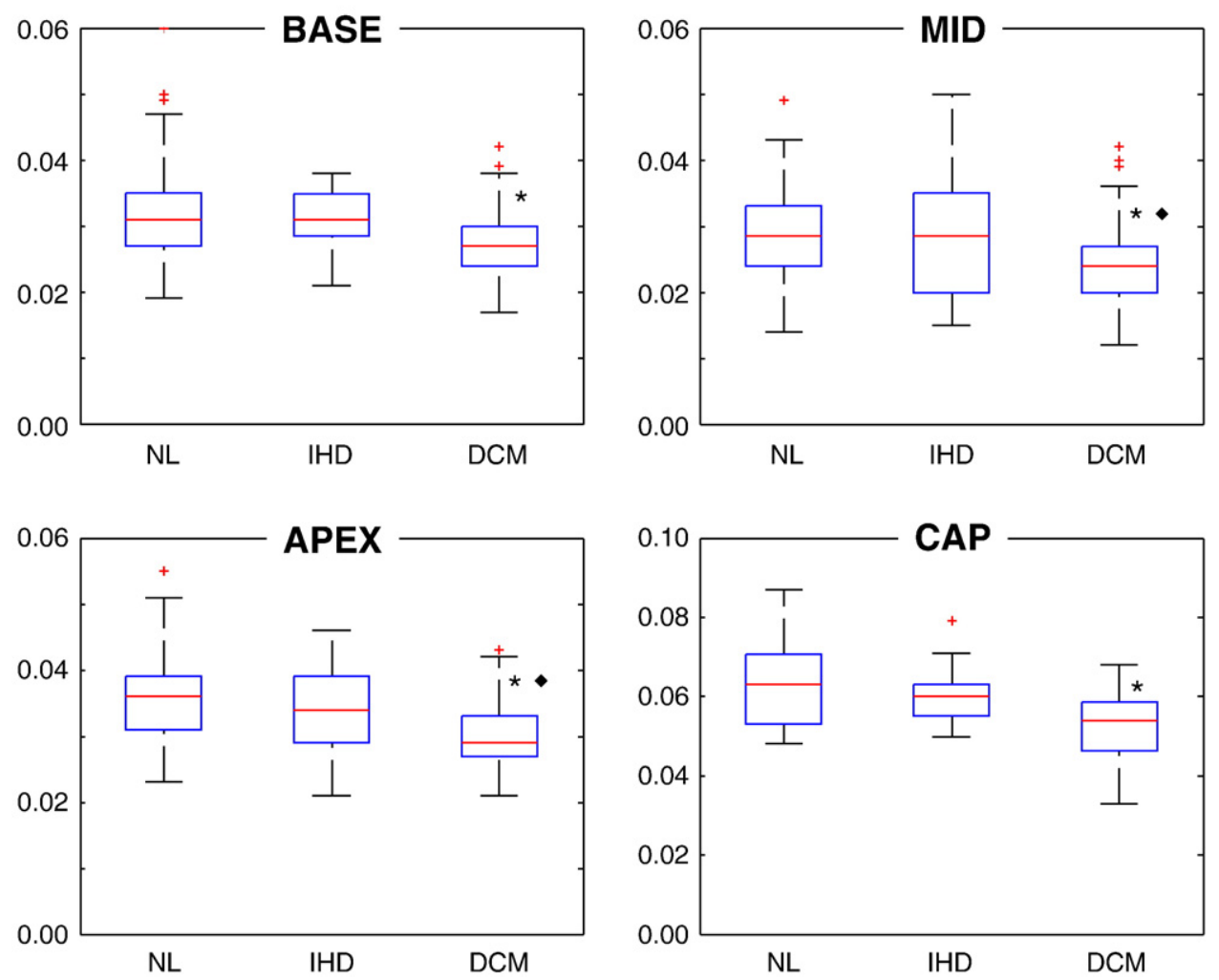

Fig. 5. Box plots of minimum regional LV endocardial curvedness at different levels of the left ventricle: comparison between the 401 normal segments (NL), 92 segments affected by ischemic heart disease (IHD) and 255 segments in patients with dilated cardiomyopathy (DCM). Values expressed in $\mathrm{m}^{-1}$ for $\mathrm{C} .{ }^{*} P<.05$ vs. NL; ${ }^{\star} P<.05$ vs. IHD; Kruskal-Wallis test. 


\section{$\underline{\operatorname{Max} C}$}
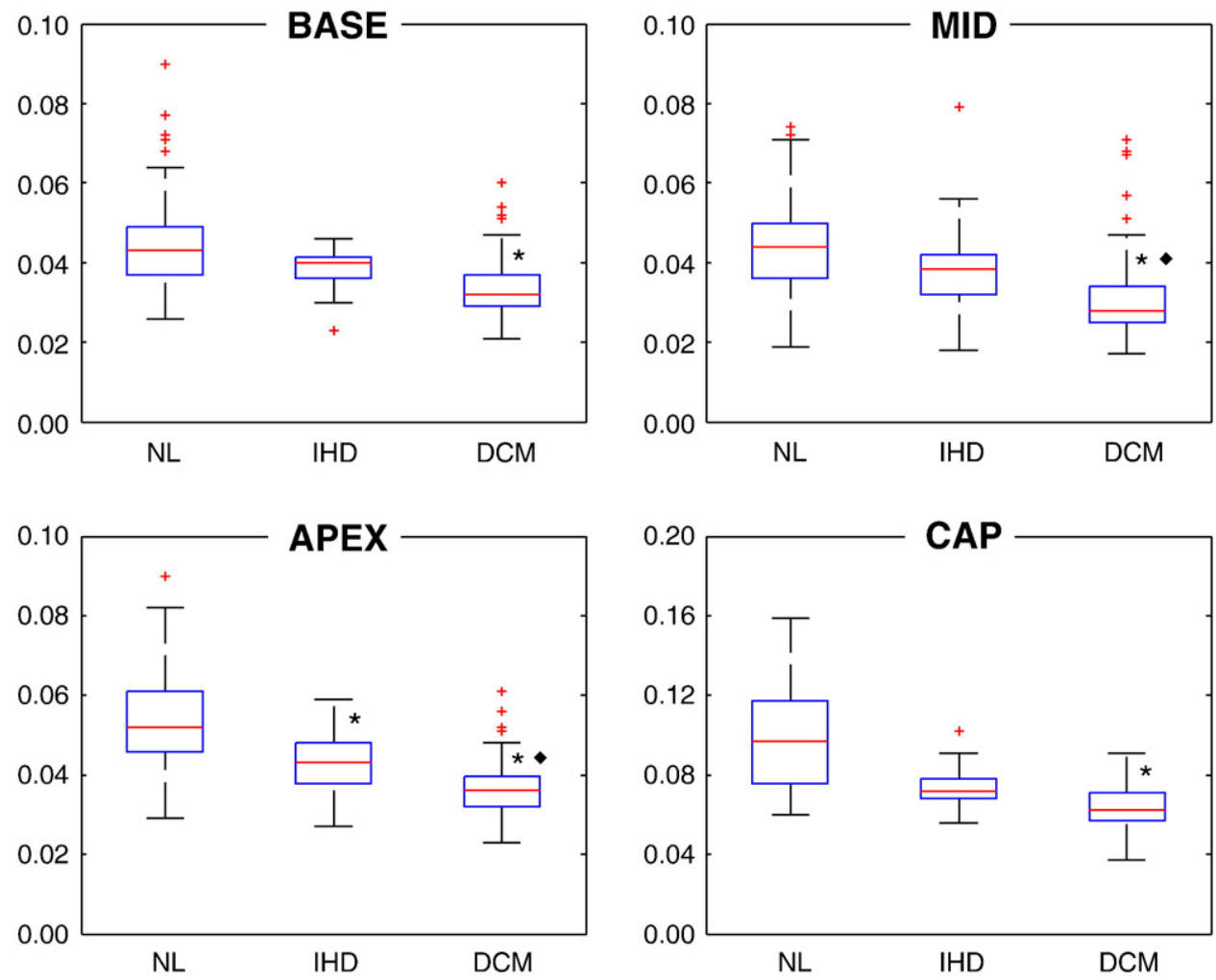

Fig. 6. Box plots of maximum regional LV endocardial curvedness at different levels of the left ventricle: comparison between 401 normal segments (NL), 92 segments affected by ischemic heard disease (IHD) and 255 segments in patients with dilated cardiomyopathy (DCM). Values expressed in $\mathrm{m}^{-1}$ for $\mathrm{C} .{ }^{*} P<.05$ vs. NL; $P<.05$ vs. IHD; Kruskal-Wallis test.

While our findings in normal ventricles demonstrating a systolic increase in endocardial curvature are expected and consistent with those previously demonstrated in a smaller group of subjects [17], our results confirmed the ability of this analysis to accurately detect subtle changes occurring frame-by-frame throughout the cardiac cycle (Fig. 3). Similarly, the increase in endocardial curvature toward the apex (Fig. 4) is intuitive, but our results have proven the ability of this analysis technique to quantify these regional differences.

The reduced maximum regional endocardial curvature in DCM (Figs. 5 and 6) is also expected and consistent with previous findings [18]. Since dilated ventricles are known to be more spherical than normal ventricles on a global level, and because ventricular dilatation in these patients is concentric and relatively uniform across segments, one could expect to obtain similar findings on a regional level given the availability of appropriate analysis tools.

Interestingly, DCM patients showed minimum value of normalized curvedness similar to the other two study groups, while maximum values were increased. Since minimum values of $C_{\mathrm{n}}$ occur at end-diastole while maximum values are end-systolic, this finding is likely due to the expected reduced LV function and not only due to an increase in $\mathrm{LV}$ volume. Importantly, the normalization step allowed separating the effects of LV volume changes from the morphologic information. Thus, the information obtained using this methodology is not redundant but rather complimentary to the standard LV volume analysis.

The reduced percent change in endocardial curvature in segments that are hypokinetic as a result of either concentric dilatation or IHD (Fig. 7) is also an expected finding consistent with reduced function in these segments irrespective of etiology. Importantly, these results have proven the ability of this technique to provide quantitative information not only in normal ventricles, but also under pathological conditions, thus providing support for clinical feasibility of this new methodology.

One limitation of our technique is that it relies on the availability of a reconstructed LV endocardial surface, which is based on subjective multiplane and multiphase initialization, tracing and corrections, and is thus prone to considerable intermeasurement variability. In this study, we used commercial software for 3D surface reconstruction and optimized the workflow to avoid misregistration of short-axis slices by initializing and optimizing endocardial boundaries in the long-axis views. We did not test the reproducibility of endocardial surface reconstruction using 


\section{Percent change in C}
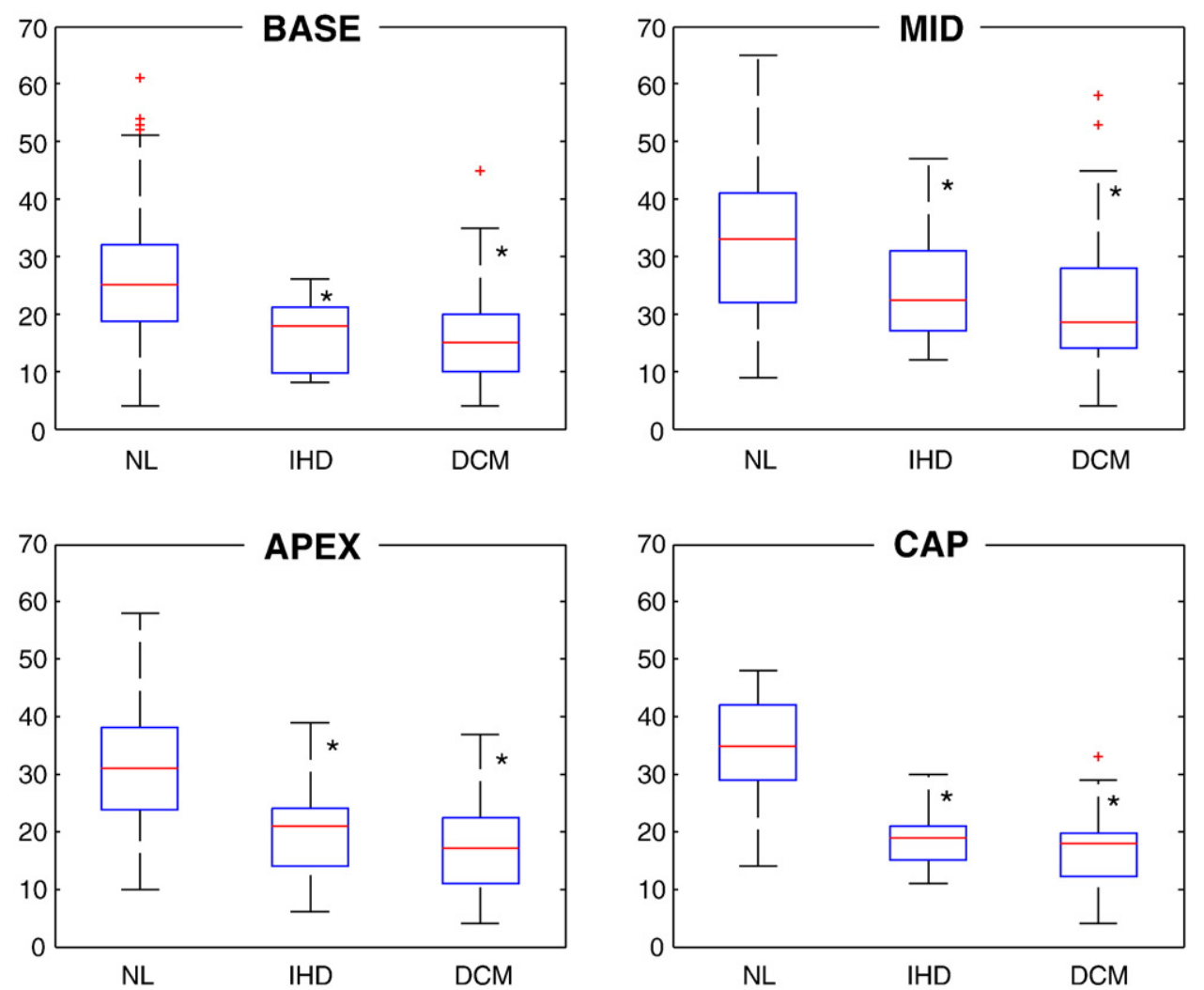

Fig. 7. Box plots of percent change in regional LV endocardial curvedness at different levels of the left ventricle: comparison between 401 normal segments (NL), 92 segments affected by ischemic heard disease (IHD) and 255 segments in patients with dilated cardiomyopathy (DCM). ${ }^{*} P<.05$ vs. NL; ${ }^{\star} P<.05$ vs. IHD; Kruskal-Wallis test.

this commercial software since this study focused on the 3D analysis of regional endocardial curvature, which is fully automated once the endocardial surface is defined, and is therefore $100 \%$ reproducible.

Importantly, this study constitutes one of the first attempts to objectively describe regional LV shape and deformation based on 3D analysis of CMR images and test the clinical feasibility of this new methodology in a relatively large number of patients with different patterns of wall motion. The ability to quantify regional LV shape may prove especially important in clinical scenarios, in which LV shape is affected locally and thus the conventional global measures of LV volume and shape may fail to differentiate normal from abnormal ventricles. One example of such common pathology would be the paradoxical septal motion in patients with right ventricular volume overload secondary to pulmonary hypertension, which is easily visualized but has been difficult to measure quantitatively. Our results indicate that this approach provides quantitative information on regional ventricular function, which is consistent with the known pathophysiology, and may thus prove clinically useful in the evaluation of LV remodeling. These results warrant future studies aimed at further clinical testing and validation in other disease states.
Supplementary materials related to this article can be found online at doi:10.1016/j.mri.2010.11.002.

\section{References}

[1] Lang RM, Bierig M, Devereux RB, Flachskampf FA, Foster E, Pellikka PA, et al. Recommendations for chamber quantification: a report from the American Society of Echocardiography's Guidelines and Standards Committee and the Chamber Quantification Writing Group, developed in conjunction with the European Association of Echocardiography, a branch of the European Society of Cardiology. J Am Soc Echocardiogr 2005; 18:1440-63.

[2] Chan J, Jenkins C, Khafagi F, Du L, Marwick TH. What is the optimal clinical technique for measurement of left ventricular volume after myocardial infarction? A comparative study of 3-dimensional echocardiography, single photon emission computed tomography, and cardiac magnetic resonance imaging. J Am Soc Echocardiogr 2006;19: 192-201.

[3] Raman SV, Shah M, McCarthy B, Garcia A, Ferketich AK. Multidetector row cardiac computed tomography accurately quantifies right and left ventricular size and function compared with cardiac magnetic resonance. Am Heart J 2006;151:736-44.

[4] Sutton M, Plappert T, Spiegel A, Raichlen J, Douglas P, Reichek N, et al. Early postoperative changes in left ventricular chamber size, architecture, and function in aortic stenosis and aortic regurgitation and their relation to intraoperative changes in afterload: a prospective two-dimensional echocardiographic study. Circulation 1987;76: $77-89$. 
[5] Pfeffer MA, Braunwald E. Ventricular remodeling after myocardial infarction. Experimental observations and clinical implications. Circulation 1990;81:1161-72.

[6] Harjai KJ, Edupuganti R, Nunez E, Turgut T, Scott L, Pandian NG. Does left ventricular shape influence clinical outcome in heart failure? Clin Cardiol 2000;23:813-9.

[7] Opie LH, Commerford PJ, Gersh BJ, Pfeffer MA. Controversies in ventricular remodelling. Lancet 2006;367:356-67.

[8] Gibson DG, Brown DJ. Continuous assessment of left ventricular shape in man. Br Heart J 1975;37:904-10.

[9] Kass DA, Traill TA, Keating M, Altieri PI, Maughan WL. Abnormalities of dynamic ventricular shape change in patients with aortic and mitral valvular regurgitation: assessment by Fourier shape analysis and global geometric indexes. Circ Res 1988;62:127-38.

[10] Azhari H, Beyar R, Sideman S. On the human left ventricular shape. Comput Biomed Res 1999;32:264-82.

[11] Azhari H, Sideman S, Beyar R, Grenadier E, Dinnar U. An analytical descriptor of three-dimensional geometry: application to the analysis of the left ventricle shape and contraction. IEEE Trans Biomed Eng 1987;34:345-55.

[12] Chan SY, Mancini GB, Fu Y, O’Brien DW, Armstrong PW. Novel methodology for echocardiographic quantification of cardiac shape. Can J Cardiol 1997;13:153-9.

[13] Di Donato M, Dabic P, Castelvecchio S, Santambrogio C, Brankovic J, Collarini $\mathrm{L}$, et al. Left ventricular geometry in normal and post-anterior myocardial infarction patients: sphericity index and 'new' conicity index comparisons. Eur J Cardiothorac Surg 2006;29(Suppl 1): S225-30.
[14] Patel AR, Lima C, Parro A, Arsenault M, Vannan MA, Pandian NG. Echocardiographic analysis of regional and global left ventricular shape in Chagas' cardiomyopathy. Am J Cardiol 1998;82:197-202.

[15] Mancini GB, DeBoe SF, Anselmo E, Simon SB, LeFree MT, Vogel RA. Quantitative regional curvature analysis: an application of shape determination for the assessment of segmental left ventricular function in man. Am Heart J 1987;113:326-34.

[16] Maffessanti F, Lang RM, Corsi C, Mor-Avi V, Caiani EG. Feasibility and clinical potential of left ventricular shape analysis from real-time 3D echocardiographic images. Ultrasound Med Biol 2009;35:1953-62.

[17] Yeo SY, Zhong L, Su Y, Tan RS, Ghista DN. A curvature-based approach for left ventricular shape analysis from cardiac magnetic resonance imaging. Med Biol Eng Comput 2009;47:313-22.

[18] Zhong L, Su Y, Yeo SY, Tan RS, Ghista DN, Kassab G. Left ventricular regional wall curvedness and wall stress in patients with ischemic dilated cardiomyopathy. Am J Physiol Heart Circ Physiol 2009;296:H573-84.

[19] Garimella RV, Swartz BK. Curvature estimation for unstructured triangulations of surfaces. Los Alamos, New Mexico: Los Alamos National Library; 2003.

[20] Koenderink JJ, van Doorn AJ. Surface shape and curvature scales. Image Vision Comput 1992;10:557-65.

[21] Cerqueira MD, Weissman NJ, Dilsizian V, Jacobs AK, Kaul S, Laskey WK, et al. Standardized myocardial segmentation and nomenclature for tomographic imaging of the heart: a statement for healthcare professionals from the Cardiac Imaging Committee of the Council on Clinical Cardiology of the American Heart Association. Circulation 2002;105:539-42. 
Video clip 1. Beating curvedness cast. 